\title{
Amino acid transporter $\mathrm{B}^{0} \mathrm{AT} 1$ influence on ADAM17 interactions with SARS-CoV-2 receptor ACE2 putatively expressed in intestine, kidney, and cardiomyocytes
}

\author{
Jacob T. Andring ${ }^{1}$, Robert McKenna ${ }^{1}$, Bruce R. Stevens ${ }^{2, *}$
}

${ }^{1}$ Department of Biochemistry and Molecular Biology

College of Medicine, University of Florida

Gainesville, FL 32610-0274 USA

${ }^{2}$ Department of Physiology and Functional Genomics

College of Medicine, University of Florida

PO Box 100274, Gainesville, FL 32610-0274 USA

${ }^{*}$ Corresponding author, Bruce R. Stevens, email: stevensb@ufl.edu

\section{ABSTRACT}

SARS-CoV-2 exhibits significant experimental and clinical gastrointestinal, renal, and cardiac muscle tropisms responsible for local tissue-specific and systemic pathophysiology capriciously occurring in about half of COVID-19 patients. The underlying COVID-19 mechanisms engaged by these extra-pulmonary organ systems are largely unknown. We approached this knowledge gap by recognizing that neutral amino acid transporter $\mathrm{B}^{0} \mathrm{AT} 1$ (alternately called NBB, B, $\mathrm{B}^{0}$ in the literature) is a common denominator expressed nearly exclusively by three particular cell types: intestinal epithelia, renal proximal tubule epithelium, and cardiomyocytes. $\mathrm{B}^{0} \mathrm{AT} 1$ provides uptake of glutamine and tryptophan. The gut is the main depot expressing over $90 \%$ of the body's entire pool of SARS-CoV-2 receptor angiotensin converting enzyme-2 (ACE2) and $\mathrm{B}^{0} \mathrm{AT} 1$. Recent cryo-EM studies established that ACE2 forms a thermodynamically favored dimer-of-heterodimers complex with $\mathrm{B}^{0} \mathrm{AT} 1$ assembled in the form of a dimer of two ACE2: $\mathrm{B}^{0} \mathrm{AT} 1$ heterodimers anchored in plasma membranes. Prior epithelial cell studies demonstrated ACE2 chaperone trafficking of $\mathrm{B}^{0} \mathrm{AT} 1$. This contrasts with monomeric expression of ACE2 in lung pneumocytes, in which $\mathrm{B}^{0} \mathrm{AT} 1$ is undetectable. The cell types in question also express a disintegrin and metalloproteinase-17 (ADAM17) known to cleave and shed the ectodomain of monomeric ACE2 from the cell surface, thereby relinquishing protection against unchecked renin-angiotensin-system (RAS) events of COVID-19. The present study employed molecular docking modeling to examine the interplaying assemblage of ACE2, ADAM17 and $\mathrm{B}^{0} \mathrm{AT} 1$. We report that in the monomer form of ACE2, neck region residues R652-N718 provide unimpeded access to ADAM17 active site pocket, but notably R708 and S709 remained $>10-15 \AA$ distant. In contrast, interference of ADAM17 docking to ACE2 in a dimer-of-heterodimers arrangement was directly correlated with the presence of a neighboring $\mathrm{B}^{0} \mathrm{AT} 1$ subunit complexed to the partnering ACE2 subunit of the $\left.2 \mathrm{ACE} 2: 2 \mathrm{~B}^{0} \mathrm{AT} 1\right]$ dimer of heterodimers, representing the expression pattern putatively exclusive to intestinal, renal and cardiomyocyte cell types. The monomer and dimer-of-heterodimers docking models were not influenced by the presence of SARS-CoV-2 receptor binding domain (RBD) complexed to ACE2. The results collectively provide the underpinnings for understanding the role of $\mathrm{B}^{0} \mathrm{AT} 1$ involvement in COVID-19 and the role of ADAM17 steering ACE2 events in intestinal and renal epithelial cells and cardiomyocytes, with implications useful for consideration in pandemic public hygiene policy and drug development. 


\section{INTRODUCTION}

While lung pathophysiology is the hallmark of COVID-19, the SARS-CoV-2 virus exhibits significant experimental and clinical gastrointestinal (GI), renal, and cardiac muscle tropisms that invoke specific local and systemic inflammasome pathophysiology [1-7]. These extra-pulmonary engagements occur capriciously in about half of COVID-19 cases, often appearing before fever onset, and extending after pulmonary symptoms abate [113]. The underlying mechanisms of COVID-19 involving these three cell types is largely unknown.

We approached this knowledge gap by recognizing that the amino acid transporter $\mathrm{B}^{0} \mathrm{AT} 1$, serving such substrates as glutamine and tryptophan, is a common denominator uniquely expressed in the plasma membranes of epithelial cells of intestine and renal proximal tubule and in cardiomyocytes, but is undetectable in lung pneumocytes [1-7, 10, 14-36]. The human GI tract is the body's site of greatest magnitude expression of both $\mathrm{B}^{0} \mathrm{AT} 1$ and the angiotensin converting enzyme-2 (ACE2) receptor for SARS-CoV-2, based on expression patterns from single cell RNA seq, immunohistochemistry, and functional genomics studies [10, 14-19, 37]. These cell types also express a disintegrin and metalloproteinase-17 (ADAM17), a key player in the landscape of COVID-19 pathophysiology $[1,2]$. The present study addresses the interplaying triangulation of these three proteins.

$\mathrm{B}^{0} \mathrm{AT} 1$ was originally discovered and functionally characterized by Stevens and coworkers [20-27] as the $\mathrm{Na}^{+}$-coupled neutral amino acid transport system in intestinal epithelial apical brush border membranes (alternately called $\mathrm{NBB}, \mathrm{B}, \mathrm{B}^{0}$ in the literature [21]), then subsequently Ganaphathy, Broer, Fairweather, Verrey, and colleagues [28-34, 38] cloned its SLC6A19 gene leading to experimental evidence of trafficking/chaperoning of $\mathrm{B}^{0} \mathrm{AT} 1$ by ACE2 to the cell surface. Building from these studies, Yan and coworkers in Zhou's lab [35] utilized cryoelectron microscopy to determine a $2.9 \AA$ complex of $\mathrm{B}^{0} \mathrm{AT} 1$ with ACE2, and established that in the presence of $\mathrm{B}^{0} \mathrm{AT} 1$, ACE2 forms a thermodynamically favored dimer-of-heterodimers complex assembled as two ACE2: $\mathrm{B}^{0} \mathrm{AT} 1$ heterodimers anchored in plasma membranes.

In contrast to $\mathrm{B}^{0} \mathrm{AT} 1$-expressing cells, lung pneumocyte membranes lack $\mathrm{B}^{0} \mathrm{AT} 1$ and express post-translationally mature $\mathrm{ACE} 2$ as a stand-alone monomer [1,2]. Regardless of the organ - whether in lung, cardiopulmonary, renal or gastrointestinal tissues - the ACE2 membrane-tethered ectodomain possesses peptidyl carboxypeptidase activity that hydrolyzes nutritive and bioactive peptides, notably angiotensin II (AngII), thus serving as protection against overactivity of the pernicious arm of the renin-angiotensin-system (RAS) [1-7, 10, 12, 14-36, 39-52]. SARS-CoV-2 hijacks the membraneattached ACE2 ectodomain as its receptor and staging arena for priming the spike S-protein leading to virion cell entry $[1,2]$.

ADAM17 is a membrane-anchored enzyme that is central to mediating a variety of proinflammatory and signaling events in epithelial and endothelial cell types. ADAM17 possesses a metzincin-like HELGHNFGAEHD active site motif that hydrolyzes nearly 100 different substrates [53]. This serves a "sheddase" function that proteolytically releases the ectodomains of membrane-bound proteins into the extracellular milieu, with the consequence that ADAM17 activity leads to pathophysiological downsides in cardiopulmonary, GI, and renal systems [54].

ADAM17 cleaves monomeric ACE2 [47, 49, 55], resulting in separation and release of the soluble ACE2 (sACE2) ectodomain from the membrane anchor stalk, thereby relinquishing its protective roles against RAS. ADAM17 is activated by AngII, and therefore SARS-CoV-2 induces an ADAM17triggered positive feedback viscous cycle escalating pernicious AngII levels unabated by ACE2 which is progressively inhibited [12]. These events of monomeric ACE2 contribute to the morbidity and mortality of COVID-19 [1, 2].

There is no consensus in the literature regarding the specific ACE2 residues targeted by ADAM17 involved in release of the ectodomain. Putative cleavage site bonds and surface residue attraction targets have been posited to lie within a broadly-defined region spanning residues R652-I741 within the neck region $[47,49,55]$, based on biochemical analyses of in vitro expression of monomeric ACE2. The role of $\mathrm{B}^{0} \mathrm{AT} 1$ in influencing the ADAM17-ACE2 relationship is unreported. To address the many important knowledge gaps in tissue-specific manifestations of COVID-19, the present projected employed molecular docking modeling to examine ADAM17's active site pocket 
access to ACE2, and the role of $\mathrm{B}^{0} \mathrm{AT} 1$ influencing the ADAM17-ACE2 relationship.

\section{METHODS}

Online protein-protein docking software ClusPro 2.0 [56-58] was employed for docking simulations, with ADAM17 (PDB ID:3LGP chain A) designated as the ligand for each paired interaction. The active site of the catalytic domain of ADAM17 is a member of the metzincins with the HEXXHXXGXXHD motif, such that ADAM17 specifically possess a zinc atom held by $\mathrm{H} 405, \mathrm{H} 409$, H415 in conjunction with glutamate at E406 serving an acid/base catalytic function[53]; therefore these four residues were chosen as ADAM17 docking "attraction" residues. Targets included PDB ID: $6 \mathrm{M} 17$ [35] employed as a $2 \mathrm{ACE} 2: 2 \mathrm{~B}^{0} \mathrm{AT} 1$ dimer-ofheterodimers complexed with SARS-CoV-2 receptor binding domain (RBD), and PDB:6M18 [35] employed analogously lacking SARS-CoV-2 RBD. Additional targets included ACE2 monomers and 1ACE2:1B ${ }^{0} \mathrm{AT} 1$ heterodimers derived from these structures using PyMOL[59]. Prior to docking, the coordinate files were modified by removing small molecules including solvent, inhibitors, ligands, glycosylation molecules, diphosphoglycerate, and non-standard amino acids. For each docking simulation, non-physiologically relevant domains of the complexes were masked out using PyMOL [59] to prevent non-specific binding (for example, hydrophobic residues within the transmembrane domain of membrane anchors).

ClusPro docks protein pairs in a three-step system [56-58]: 1) rigid body docking placed through 70,000 rotations; 2) clustering of low energy complexes; 3) energy minimization. The docked complexes are scored based on ClusPro 2.0 equation of the general form,

$$
E=w_{1} E_{r e p}+w_{2} E_{a t t r}+w_{3} E_{\text {elec }}+w_{4} E_{D A R S}
$$

where $E_{\text {rep }}$ is energy of repulsion from van der Waals interactions, $E_{\text {attr }}$ is energy of attraction from van der Waals interactions, $E_{\text {elec }}$ is the electrostatic energy term, and $E_{D A R S}$ is the energy of desolvation. Docking scoring was based on the "Balanced" option choice with constants $w_{1}=0.4, w_{2}=-0.4, w_{3}=600$, and $w_{4}$ $=1.0$. ClusPro clusters together the top scored 1000 docked positions by finding a local center with the most docking neighbors within a $9 \AA$ sphere [58]. Energy minimization within a given cluster is used to remove any steric clashes between side chains, with the top docking scores outputted along with the corresponding complexed structure coordinate files $[57,58]$. The best model chosen from among the output options resulting from each ClusPro docking was based on the highest ranked most populated cluster center (cluster size), rather than based on individual lowest energy structures, as recommended by Vajda and colleagues and CAPRI experiment data [56-58]. PDBePISA [60] was used to determine the binding contact interface residues. Figures were generated using PyMOL[59].

\section{RESULTS}

The fundamental ACE2 monomer organization $[1,2]$ is shown in Fig. 1A, whereby a hydrophobic transmembrane stalk within the plasma membrane anchors and tethers the ACE2 ectodomain to the cell surface via a neck region. Molecular modeling results of the present study collectively implicated a span of ACE2 residues covering R652N718 within the neck region (Fig. 1B) that provided the target for attempted dockings by ADAM17 as the ligand. In the case of monomeric ACE2, Fig. 2 shows the successful docking model, whereby ADAM17 catalytic zinc atom and its corresponding active site pocket residues H415 and E406 were attracted $\leq 2-3$ $\AA$ to ACE2 residues R652, K657 and K659, but notably $>10-15 \AA$ distant to ACE R708 and S709.

In contrast to monomer ACE2, Fig. 3 shows that ADAM17 active site pocket residues failed to dock appropriately with the dimer-of-heterodimers comprised of two ACE2 subunits complexed with two $\mathrm{B}^{0} \mathrm{AT} 1$ subunits; the results were the same whether the ACE2 subunits were complexed to SARS-CoV-2 RBD (6M18, Fig. 3A,B) or excluding SARS-CoV-2 RBD (6M17, Fig. 3C,D). In either scenario, docking attempts resulted in ADAM17 active site pocket residues exceeding $>10-15 \AA$ to ACE2 neck region representative residues ACE2_R652, ACE2_K657, or ACE2_K659.

To explore the nature of the interferences observed in Fig. 3, ADAM17 ligand dockings were then attempted by employing targets comprised of a single heterodimer comprised of $1 \mathrm{ACE} 2: 1 \mathrm{~B}^{0} \mathrm{AT} 1$, as shown in Fig. 4. Here, ADAM17 active site residues made successful docking contacts at distances $\leq 2-3$ $\AA$ with neck region residues of heterodimer 1ACE2:1B ${ }^{0} \mathrm{AT} 1$, either without SARS-CoV-2 RBD (Fig. 4A,B) or with RBD (Fig. 4C,D) complexed to the ACE2 subunit, with the exception that 
ACE2_R708 and ACE2_S709 remained distant. The data of Figs. 3 and 4 were then assembled to reveal the influence of $\mathrm{B}^{0} \mathrm{AT} 1$ on the superposition of ADAM17 docking in relation to ACE2 neck region residues ACE2_R652, ACE_K657, ACE2_K659,
R708, and S709 as shown in Fig. 5. The results (Figs. 3A-D and 5A) collectively implicated ADAM17 docking interference as assigned to the presence of the second $\mathrm{B}^{0} \mathrm{AT} 1$ complexed to the neighboring ACE2 of the dimer-of-heterodimers assembly.
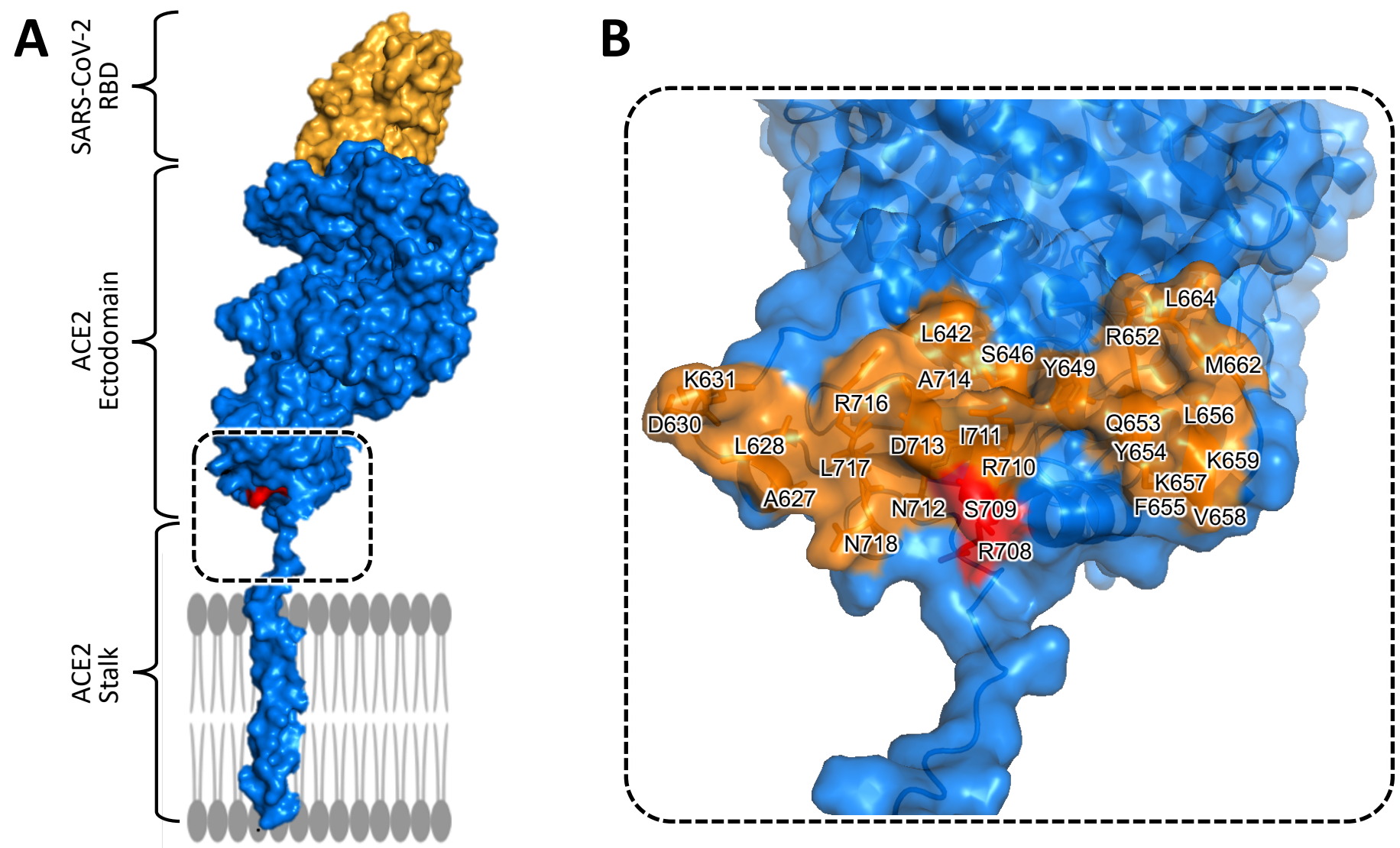

Fig. 1. ACE2 monomer with neck region residues bridging ectodomain to anchor.

A. ACE2 monomer (blue) shown with hydrophobic membrane anchoring stalk, and with the ectodomain complexed with SARS-CoV-2 receptor binding domain (RBD, gold). Box denotes ACE2 neck region spanning residues R652-N718. B. Closeup of box in (A) labeled with key neck region (brown) residues spanning R652-N718, which includes $\mathrm{R} 708$ and S709 residues (red). 


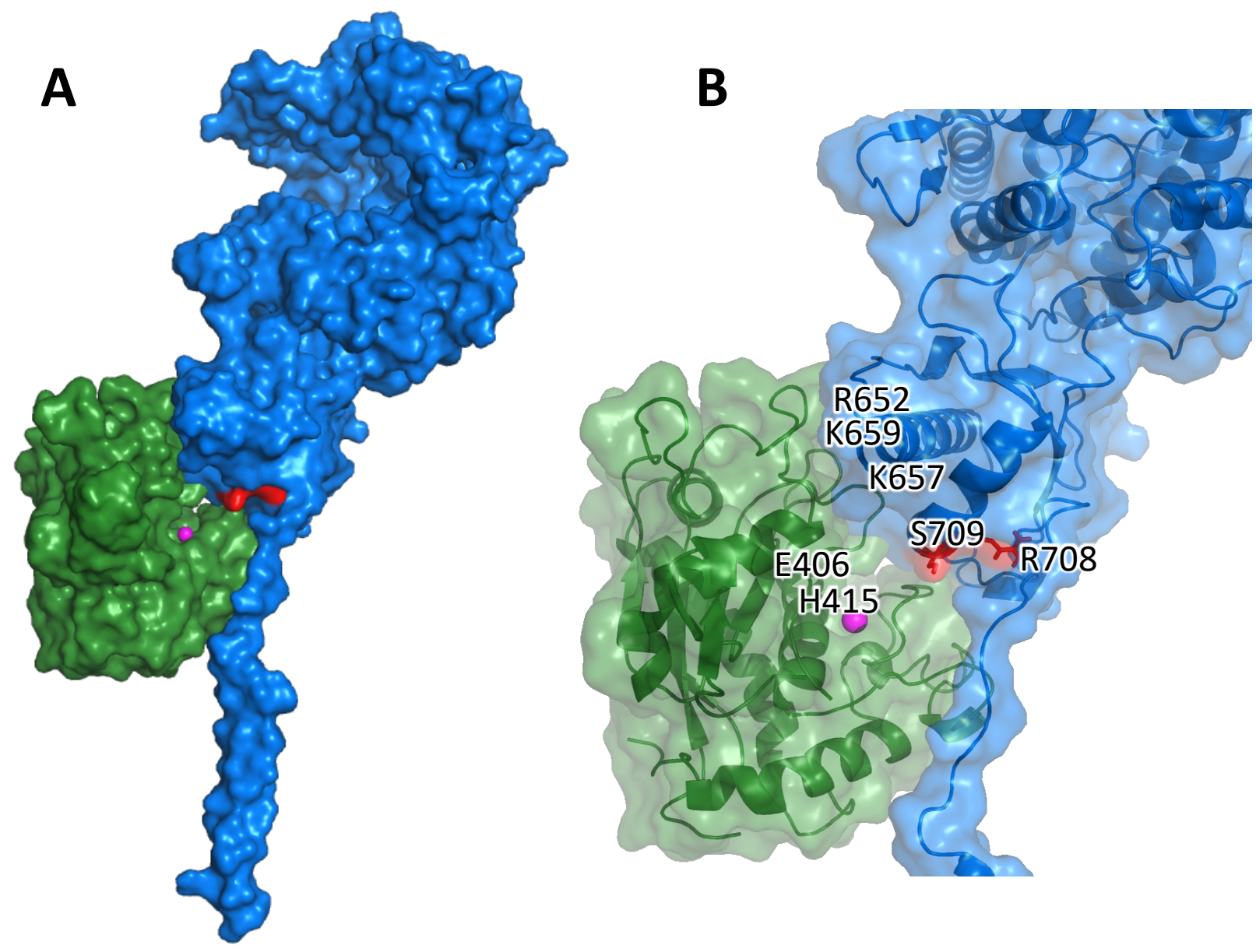

Fig. 2. ACE2 monomer target successful docking with ADAM17 ligand.

A. ADAM17 (green) ligand docking to unobstructed readily accessible monomer ACE2 (blue) neck region residues. B. Closeup of ADAM17-ACE2 docking contact interface showing ADAM17 catalytic zinc (magenta) and active site pocket residues $\mathrm{H} 415$ and E406 of ADAM17 attractions $\leq 2-3 \AA$ to ACE2 residues R652, K657 and K659, but $>10-15$ A distant to ACE2 R708 and ACE S709 (red). Similar results were obtained with SARS-CoV-2 RBD complexed to the ACE2. 

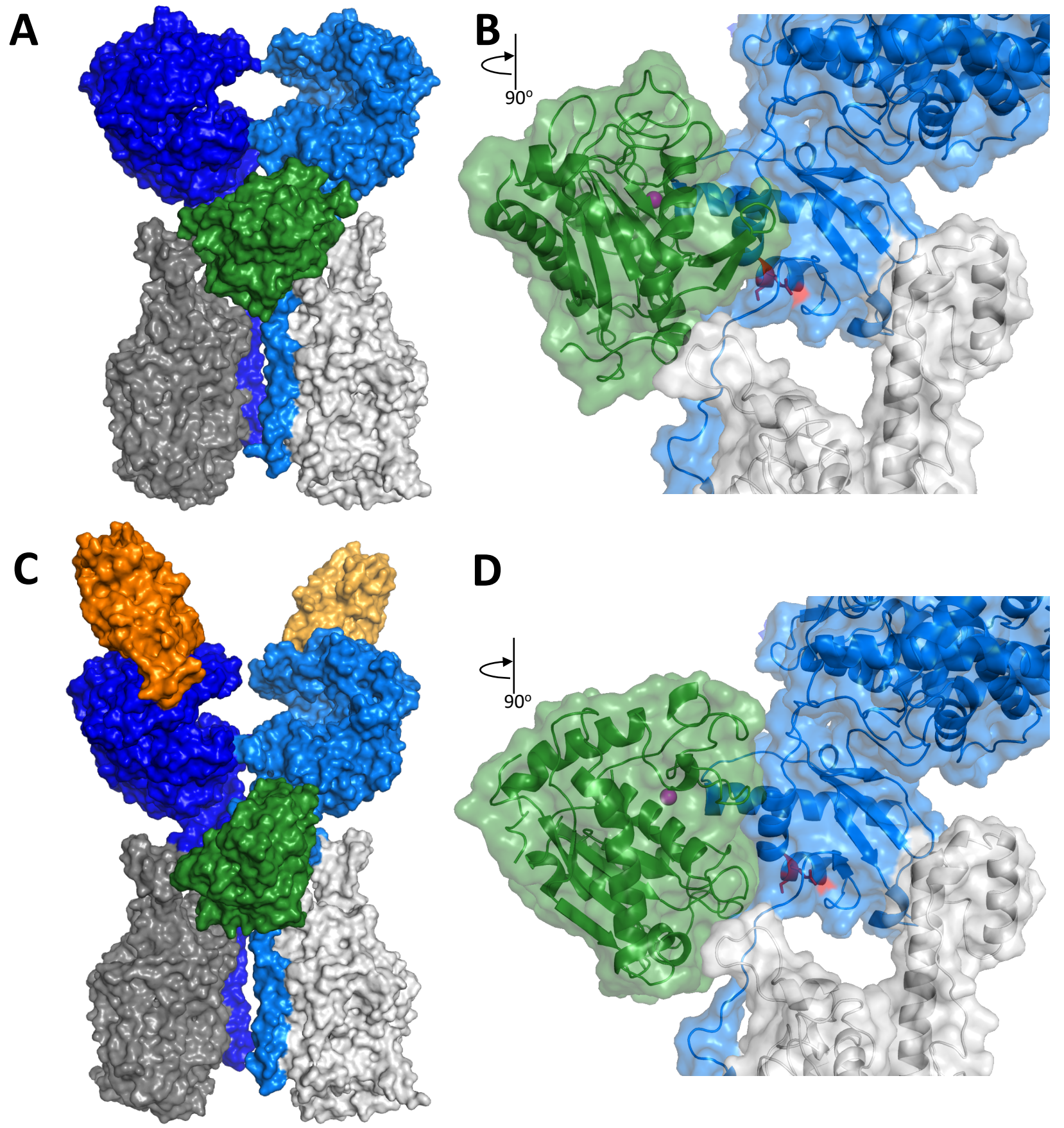

Fig. 3. Interference of ADAM17 docking with dimer-of-heterodimeric $2 A C E 2: 2 B^{0} A T 1$ complexes $6 M 17$ or $6 \mathrm{M} 18$.

A. Front view of failed attempted docking contact of ADAM17 (green) ligand with PDB ID: 6 M18 dimer-ofheterodimers comprised of two ACE2 subunits (light blue and dark blue) with two $B^{0} A T 1$ subunits (light gray and dark gray). B. Closeup side view of (A) showing ligand ADAM17 (green) catalytic pocket with zinc atom (magenta) hindered from docking contact ( $>10-15 \AA$ distance) with ACE2 neck region residues. C. Front view of failed attempted docking contact of ADAM17 with PDB ID: 6M17 dimer-of-heterodimers comprised of two $\mathrm{B}^{0} \mathrm{AT} 1$ subunits with two ACE2 subunits complexed with SARS-CoV-2 RBD (gold and brown). D. Closeup side view of (C) showing ligand ADAM17 (green) catalytic pocket with zinc atom (magenta) occluded from docking contact (>10-15 Å distance) with ACE2 neck region residues. 

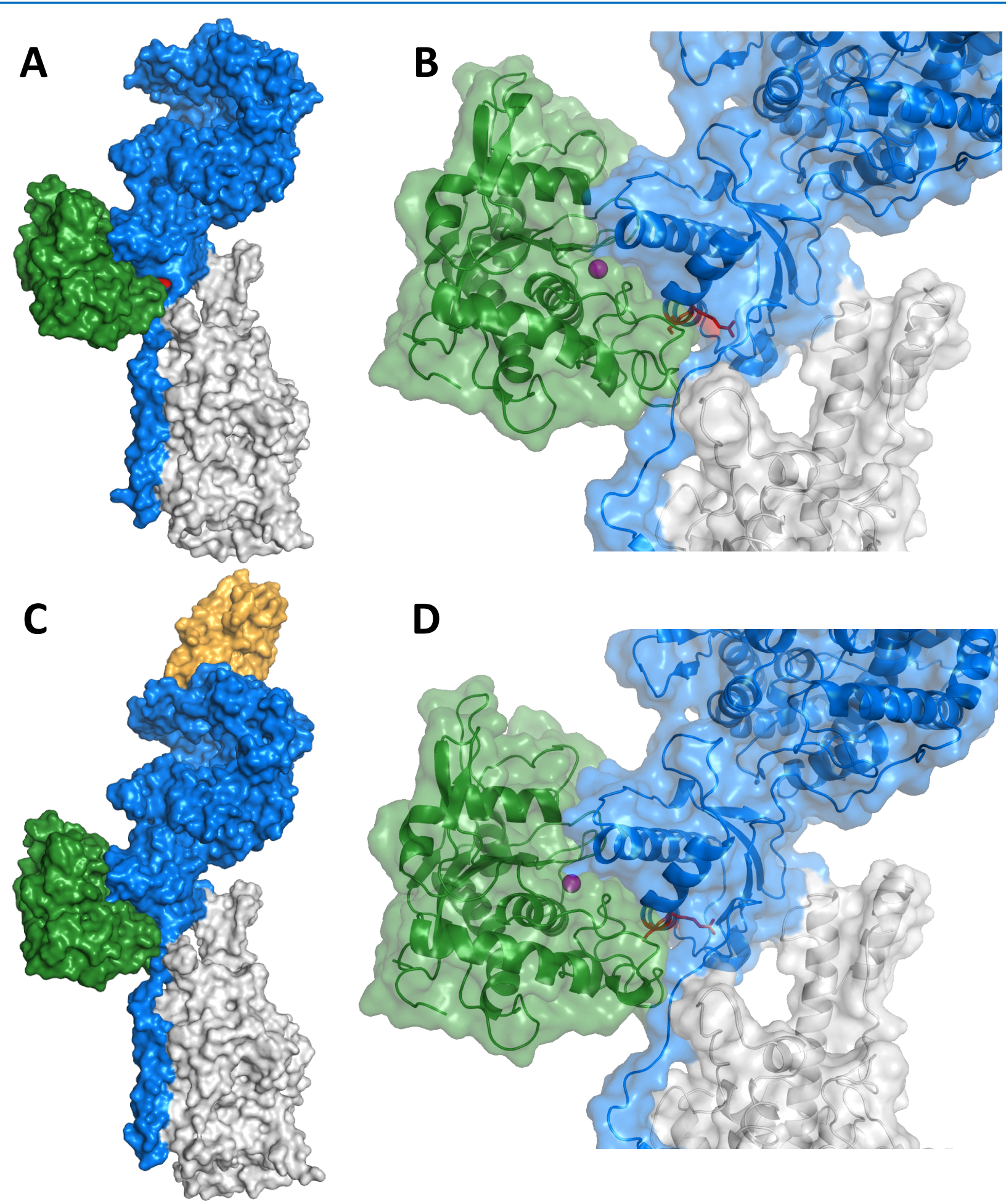

Fig. 4. ADAM17 successful docking to $1 A C E 2: 1 B^{0} A T 1$ heterodimer.

A. ADAM17 (green) ligand successful docking contact with heterodimer of 1 ACE2 (blue):1B ${ }^{0}$ AT1 (gray). B. Closeup view of (A) showing ADAM17 (green) active site pocket residues (with zinc atom (magenta) docked $(\leq 2-3 \AA)$ to $1 \mathrm{ACE} 2: 1 \mathrm{~B}^{0} \mathrm{AT} 1$ heterodimer complex. C. ADAM17 successful docking to $1 \mathrm{ACE} 2: 1 \mathrm{~B}^{0} \mathrm{AT} 1$ complexed with SARS-CoV-2 RBD (gold) similar to (A). D. Closeup view of (C) showing docking contact results the same as (B). Although these $1 A C E 2: 1 B^{0} A T 1$ heterodimers do not have physiological antecedence with experimental evidence, these dockings were included for completeness of the combination permutations of $A C E 2$ and $B^{0} A T 1$ subunits. 

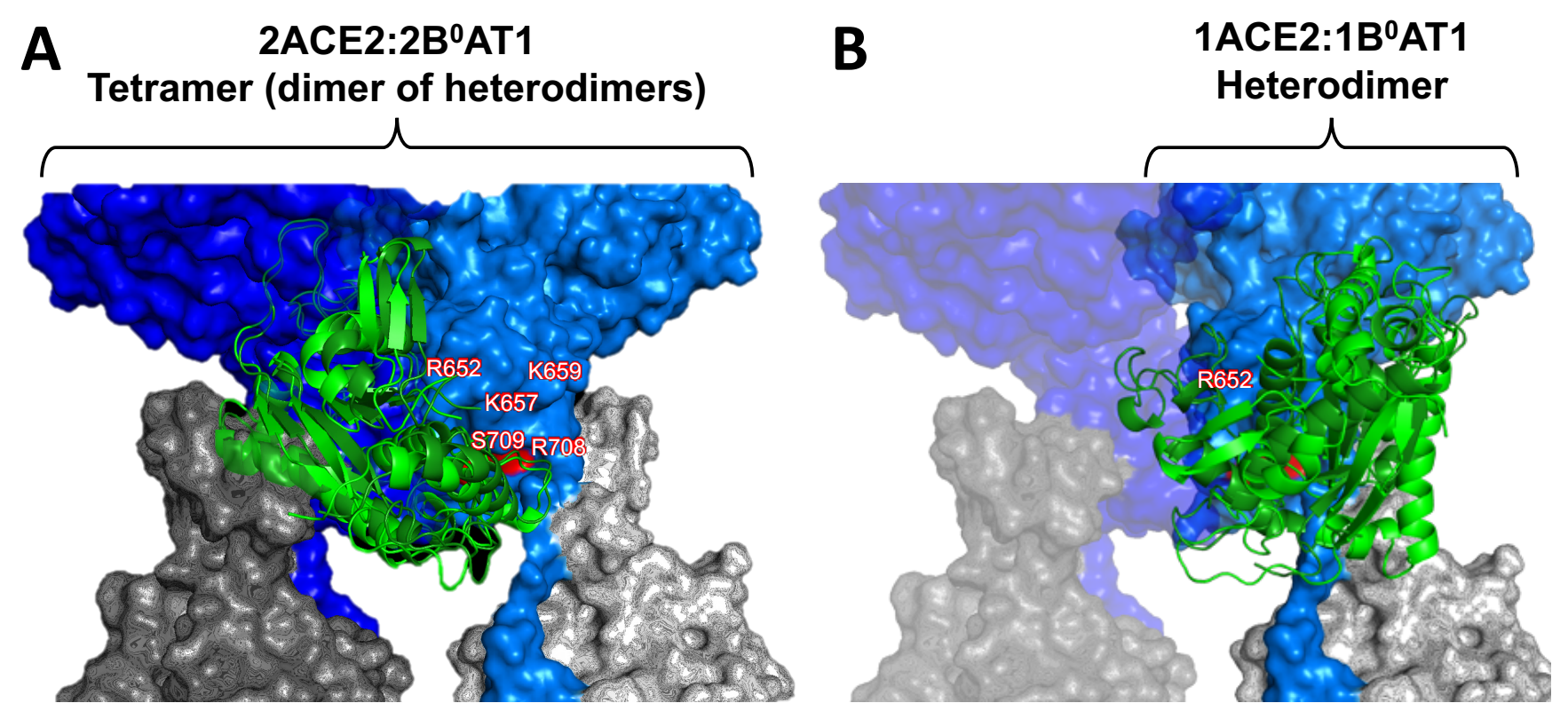

Fig. 5. Superposition of ADAM17 docking.

A. Interference of ADAM17 docking to 2ACE2:2B ${ }^{0}$ AT1 dimer-of-heterodimers (PDB ID:6M18 or 6M17). Presence of neighboring $B^{0} A T 1$ subunit (dark gray at left of $A$ ) of partner ACE2 (dark blue at left of $A$ ) interfered with ADAM17 (green) successful docking contact with neck residues of the other attached ACE2 (light blue at right of $A$ complexed with partner $B^{0} A T 1$ subunit, light gray at right of $A$ ). ACE2 neck residues are representatively shown located at ACE2_R652, ACE_K657, ACE2_K659, ACE2_R708 and S709 each >10 ̊ distance to ADAM17 active site pocket residues.

B. Successful docking of ADAM17 to $1 A C E 2: 2 B^{0} A T 1$ heterodimer. Heterodimer components are shown in opaque colors, with transparent ghost of neighboring chains of full dimer-of-heterodimer shown for steric orientation relative to panel (A). Here, ACE2_R652, ACE_K657, ACE2_K659 were $\leq 3 \AA$ from ADAM17, while ACE2_R708 and ACE2_S709 remained >10 Å distant. 


\section{DISCUSSION}

The main findings of this in silico molecular docking modeling study are: 1) ADAM17 active site pocket can unimpededly dock and form interface contacts with neck region residues of monomer ACE2, yet the ADAM17 active site remains distant from ACE2_R708 and ACE2_S709; and 2) interference of ADAM17 docking to ACE2 in dimerof-heterodimer arrangements was directly correlated with the presence of a neighboring $\mathrm{B}^{0} \mathrm{AT} 1$ subunit that is complexed to the partnering ACE2 subunit of the $2 \mathrm{x}$ [ACE2: $\mathrm{B}^{0} \mathrm{AT} 1$ ] dimer-of-heterodimers-with or without SARS-CoV-2 RBD-representing the expression pattern putatively exclusive to intestinal, renal and cardiomyocyte cell types [1-7]. Experimental evidence is lacking in the literature concerning interactions of ADAM17 with ACE2 in organs that exhibit SARS-CoV-2 tropism and are comprised of these cell types that express $\mathrm{B}^{0} \mathrm{AT} 1$. Although the 1ACE2:1B ${ }^{0} \mathrm{AT} 1$ heterodimer (note: not the $2 \mathrm{x}$ [ACE2: $\mathrm{B}^{0} \mathrm{AT} 1$ ] dimer-of-heterodimers) does not have physiological antecedence in the literature, the heterodimer molecular dockings were included in the present project for completeness of the permuted combinations of ACE2 with $\mathrm{B}^{0} \mathrm{AT} 1$ subunits in order to arrive at the ADAM17 superposition results shown in Fig. 5.

In vitro experiments $[47,49,55]$ have posited that a span covering monomer ACE2 residues R652I741 may be involved in ADAM17 binding, resulting in the shedding of soluble ACE2 ectodomain away from the cell surface into the extracellular milieu. This was corroborated by the present modeling results that implicated ACE2 candidate residues limited to R652-N718 in its neck region (Fig. 1B). However, the literature is conflicted regarding specific roles of ACE2 neck residues in relation to ADAM17 cleavage or other ADAM17 roles such as neck region competition with alternative ACE2targeted proteases such as transmembrane serine protease-2 (TMPRSS2). Heurich and coworkers [49] employed a transgenic expression system yielding biochemical experimental data implicating ACE2 residues 652-659 as essential for binding recognition by ADAM17 or TMPRSS2. Although Heurich experiments [49] did not ascertain the exact bonds cleaved for shedding the ACE2 ectodomain, the putative cleavage site was reported to be likely somewhere within its neck span of residues 697-716, specifically excluding R621. Jia et al [55] also utilized biochemical experiments to contend that the putative cleavage could occur in the span of ACE2 residues 716-741. In another set of experiments, Lai [47] implicated cleavage between ACE2 residues R708/S709, with R710 playing a role in presumed binding recognition. The present docking modeling results (Figs. 2-5) indicate that the closest ADAM17 active site residues approached ACE2 R708 was $>10$ $\AA$ for any of the ACE2 conformations tested, whether ACE2 monomer, heterodimer, or dimer-ofheterodimers. Therefore, the present docking modeling is the most consistent with, and primarily supports, the experimental data of Heurich [49], and refutes the premise of Lai [47] that the cleavage occurs between ACE2 residues R708/S709.

In conclusion, these findings collectively provide the underpinnings and gateway to future wet lab experiments designed to understand the role of $\mathrm{B}^{0} \mathrm{AT} 1$ involvement in COVID-19 and the role of ADAM17 steering ACE2 events. This is especially important relating to the capricious manifestations of COVID-19 in intestinal and renal epithelial cells and cardiomyocytes, with implications useful for consideration in pandemic public hygiene policy and drug development.

\section{REFERENCES CITED}

1. Sharma, R.K., B.R. Stevens, A.G. Obukhov, M.B. Grant, et al., ACE2 (Angiotensin-Converting Enzyme 2) in Cardiopulmonary Diseases: Ramifications for the Control of SARS-CoV-2. Hypertension, 2020. 76(3): p. 651-661.

2. Obukhov, A.G., B.R. Stevens, R. Prasad, S. Li Calzi, et al., SARS-CoV-2 Infections and ACE2: Clinical Outcomes Linked With Increased Morbidity and Mortality in Individuals With Diabetes. Diabetes, 2020. 69(9): p. 1875-1886.

3. Zang, R., M.F. Gomez Castro, B.T. McCune, Q. Zeng, et al., TMPRSS2 and TMPRSS4 promote SARS-CoV-2 infection of human small intestinal enterocytes. Sci Immunol, 2020. 5(47).

4. Sharma, A., G. Garcia, Jr., Y. Wang, J.T. Plummer, et al., Human iPSC-Derived Cardiomyocytes Are Susceptible to SARS-CoV-2 Infection. Cell Rep Med, 2020. 1(4): p. 100052. 
5. Werion, A., L. Belkhir, M. Perrot, G. Schmit, et al., SARS-CoV-2 Causes a Specific Dysfunction of the Kidney Proximal Tubule. Kidney Int, 2020.

6. Lamers, M.M., J. Beumer, J. van der Vaart, K. Knoops, et al., SARS-CoV-2 productively infects human gut enterocytes. Science, 2020.

7. Robinson, E.L., K. Alkass, O. Bergmann, J.J. Maguire, et al., Genes encoding ACE2, TMPRSS2 and related proteins mediating SARS-CoV-2 viral entry are upregulated with age in human cardiomyocytes. J Mol Cell Cardiol, 2020. 147: p. 88-91.

8. Cholankeril, G., A. Podboy, V.I. Aivaliotis, B. Tarlow, et al., High Prevalence of Concurrent Gastrointestinal Manifestations in Patients with SARS-CoV-2: Early Experience from California. Gastroenterology, 2020.

9. Wong, S.H., R.N. Lui, and J.J. Sung, Covid-19 and the Digestive System. J Gastroenterol Hepatol, 2020.

10. Zhang, H., H.B. Li, J.R. Lyu, X.M. Lei, et al., Specific ACE2 expression in small intestinal enterocytes may cause gastrointestinal symptoms and injury after 2019-nCoV infection. Int J Infect Dis, 2020. 96: p. 19-24.

11. Parasa, S., M. Desai, V. Thoguluva Chandrasekar, H.K. Patel, et al., Prevalence of Gastrointestinal Symptoms and Fecal Viral Shedding in Patients With Coronavirus Disease 2019: A Systematic Review and Meta-analysis. JAMA Netw Open, 2020. 3(6): p. e2011335.

12. Gheblawi, M., K. Wang, A. Viveiros, Q. Nguyen, et al., Angiotensin-Converting Enzyme 2: SARSCoV-2 Receptor and Regulator of the ReninAngiotensin System: Celebrating the 20th Anniversary of the Discovery of ACE2. Circ Res, 2020. 126(10): p. 1456-1474.

13. Wong, M.C., J. Huang, C. Lai, R. Ng, et al., Detection of SARS-CoV-2 RNA in fecal specimens of patients with confirmed COVID-19: a metaanalysis. J Infect, 2020.

14. Ponten, F., K. Jirstrom, and M. Uhlen, The Human Protein Atlas--a tool for pathology. J Pathol, 2008. 216(4): p. 387-93.

15. Chen, Q.L., J.Q. Li, Z.D. Xiang, Y. Lang, et al., Localization of Cell Receptor-Related Genes of SARS-CoV-2 in the Kidney through Single-Cell
Transcriptome Analysis. Kidney Diseases, 2020(published online doi:

10.1159/000508162).

16. Human_Protein_Atlas. [Human Protein Atlas] 2020; Available from: http://proteinatlas.org.

17. Thul, P.J., L. Akesson, M. Wiking, D. Mahdessian, et al., A subcellular map of the human proteome. Science, 2017. 356(6340).

18. Zou, X., K. Chen, J. Zou, P. Han, et al., Single-cell RNA-seq data analysis on the receptor ACE2 expression reveals the potential risk of different human organs vulnerable to 2019-nCoV infection. Front Med, 2020.

19. Wrapp, D., N. Wang, K.S. Corbett, J.A. Goldsmith, et al., Cryo-EM structure of the 2019-nCoV spike in the prefusion conformation. Science, 2020. 367(6483): p. 1260-1263.

20. Pan, M. and B.R. Stevens, Differentiation- and protein kinase $C$-dependent regulation of alanine transport via system B. J Biol Chem, 1995. 270(8): p. 3582-7.

21. Stevens, B.R., Amino Acid Transport by Epithelial Membranes, in Epithelial Transport Physiology, G.A. Gerencser, Editor. 2010, Humana Press. p. 353-378.

22. Stevens, B.R., S.H. Wright, B.S. Hirayama, R.D. Gunther, et al., Organic and inorganic solute transport in renal and intestinal membrane vesicles preserved in liquid nitrogen. Membr Biochem, 1982. 4(4): p. 271-82.

23. Stevens, B.R., H.J. Ross, and E.M. Wright, Multiple transport pathways for neutral amino acids in rabbit jejunal brush border vesicles. J Membr Biol, 1982. 66(3): p. 213-25.

24. Stevens, B.R., J.D. Kaunitz, and E.M. Wright, Intestinal transport of amino acids and sugars: advances using membrane vesicles. Annu Rev Physiol, 1984. 46: p. 417-33.

25. Stevens, B., Amino acid transport in intestine., in Mammalian Amino Acid Transport, M. Kilberg and D. Haussinger, Editors. 1992, Plenum Press: New York. p. 149-163.

26. Souba, W.W., M. Pan, and B.R. Stevens, Kinetics of the sodium-dependent glutamine transporter in human intestinal cell confluent monolayers. Biochem Biophys Res Commun, 1992. 188(2): p. 746-53. 
27. Pan, M., W.W. Souba, C.L. Wolfgang, A.M. Karinch, et al., Posttranslational alanine transstimulation of zwitterionic amino acid transport systems in human intestinal Caco-2 cells. J Surg Res, 2002. 104(1): p. 63-9.

28. Broer, S., Apical transporters for neutral amino acids: physiology and pathophysiology. Physiology (Bethesda), 2008. 23: p. 95-103.

29. Camargo, S.M., D. Singer, V. Makrides, K. Huggel, et al., Tissue-specific amino acid transporter partners ACE2 and collectrin differentially interact with hartnup mutations. Gastroenterology, 2009. 136(3): p. 872-82.

30. Vuille-dit-Bille, R.N., S.M. Camargo, L. Emmenegger, T. Sasse, et al., Human intestine luminal ACE2 and amino acid transporter expression increased by ACE-inhibitors. Amino Acids, 2015. 47(4): p. 693-705.

31. Fairweather, S.J., A. Broer, N. Subramanian, E. Tumer, et al., Molecular basis for the interaction of the mammalian amino acid transporters BOAT1 and BOAT3 with their ancillary protein collectrin. J Biol Chem, 2015. 290(40): p. 2430825.

32. Hashimoto, T., T. Perlot, A. Rehman, J. Trichereau, et al., ACE2 links amino acid malnutrition to microbial ecology and intestinal inflammation. Nature, 2012. 487(7408): p. 47781.

33. Jando, J., S.M.R. Camargo, B. Herzog, and F. Verrey, Expression and regulation of the neutral amino acid transporter BOAT1 in rat small intestine. PLoS One, 2017. 12(9): p. e0184845.

34. Fairweather, S.J., A. Bröer, M.L. O'Mara, and S. Bröer, Intestinal peptidases form functional complexes with the neutral amino acid transporter B(0)AT1. Biochem J, 2012. 446(1): $p$. 135-48.

35. Yan, R., Y. Zhang, Y. Li, L. Xia, et al., Structural basis for the recognition of SARS-CoV-2 by fulllength human ACE2. Science, 2020. 367(6485): p. 1444-1448.

36. Scalise, M. and C. Indiveri, Repurposing Nimesulide, a Potent Inhibitor of the BOAT1 Subunit of the SARS-CoV-2 Receptor, as a Therapeutic Adjuvant of COVID-19. SLAS Discov, 2020: p. 2472555220934421.
37. Zhang, H., Z. Kang, H. Gong, D. Xu, et al., Digestive system is a potential route of COVID19: an analysis of single-cell coexpression pattern of key proteins in viral entry process. Gut, 2020: p. gutjnl-2020-320953.

38. Kekuda, R., V. Torres-Zamorano, Y.J. Fei, P.D. Prasad, et al., Molecular and functional characterization of intestinal $\mathrm{Na}(+)$-dependent neutral amino acid transporter BO. Am J Physiol, 1997. 272(6 Pt 1): p. G1463-72.

39. Burgueno, J.F., A. Reich, H. Hazime, M.A. Quintero, et al., Expression of SARS-CoV-2 Entry Molecules ACE2 and TMPRSS2 in the Gut of Patients With IBD. Inflamm Bowel Dis, 2020. 26(6): p. 797-808.

40. Koga, H., H. Yang, E.Q. Haxhija, and D.H. Teitelbaum, The role of angiotensin II type 1a receptor on intestinal epithelial cells following small bowel resection in a mouse model. Pediatr Surg Int, 2008. 24(12): p. 1279-86.

41. Wong, T.P., E.S. Debnam, and P.S. Leung, Involvement of an enterocyte renin-angiotensin system in the local control of SGLT1-dependent glucose uptake across the rat small intestinal brush border membrane. J Physiol, 2007. 584(Pt 2): p. 613-23.

42. Wong, T.P., K.Y. Ho, E.K. Ng, E.S. Debnam, et al., Upregulation of ACE2-ANG-(1-7)-Mas axis in jejunal enterocytes of type 1 diabetic rats: implications for glucose transport. Am J Physiol Endocrinol Metab, 2012. 303(5): p. E669-81.

43. Gembardt, F., A. Sterner-Kock, H. Imboden, M. Spalteholz, et al., Organ-specific distribution of ACE2 $\mathrm{mRNA}$ and correlating peptidase activity in rodents. Peptides, 2005. 26(7): p. 1270-7.

44. Garg, M., P.W. Angus, L.M. Burrell, C. Herath, et al., Review article: the pathophysiological roles of the renin-angiotensin system in the gastrointestinal tract. Aliment Pharmacol Ther, 2012. 35(4): p. 414-28.

45. Chan, L.K.Y. and P.S. Leung, Multifaceted interplay among mediators and regulators of intestinal glucose absorption: potential impacts on diabetes research and treatment. American Journal of Physiology-Endocrinology and Metabolism, 2015. 309(11): p. E887-E899. 
46. Lambert, D.W., M. Yarski, F.J. Warner, P. Thornhill, et al., Tumor necrosis factor-alpha convertase (ADAM17) mediates regulated ectodomain shedding of the severe-acute respiratory syndrome-coronavirus (SARS-CoV) receptor, angiotensin-converting enzyme-2 (ACE2). J Biol Chem, 2005. 280(34): p. 30113-9.

47. Lai, Z.W., I. Hanchapola, D.L. Steer, and A.I. Smith, Angiotensin-converting enzyme 2 ectodomain shedding cleavage-site identification: determinants and constraints. Biochemistry, 2011. 50(23): p. 5182-94.

48. Zunke, F. and S. Rose-John, The shedding protease ADAM17: Physiology and pathophysiology. Biochim Biophys Acta Mol Cell Res, 2017. 1864(11 Pt B): p. 2059-2070.

49. Heurich, A., H. Hofmann-Winkler, S. Gierer, T. Liepold, et al., TMPRSS2 and ADAM17 cleave ACE2 differentially and only proteolysis by TMPRSS2 augments entry driven by the severe acute respiratory syndrome coronavirus spike protein. J Virol, 2014. 88(2): p. 1293-307.

50. Singer, D. and S.M. Camargo, Collectrin and ACE2 in renal and intestinal amino acid transport. Channels (Austin), 2011. 5(5): p. 41023.

51. Garg, M., S.G. Royce, C. Tikellis, C. Shallue, et al., Imbalance of the renin-angiotensin system may contribute to inflammation and fibrosis in IBD: a novel therapeutic target? Gut, 2020. 69(5): p. 841-851.

52. Yisireyili, M., Y. Uchida, K. Yamamoto, T. Nakayama, et al., Angiotensin receptor blocker irbesartan reduces stress-induced intestinal inflammation via AT1a signaling and ACE2dependent mechanism in mice. Brain Behav Immun, 2018. 69: p. 167-179.

53. Gomis-Ruth, F.X., Catalytic Domain Architecture of Metzincin Metalloproteases. Journal of Biological Chemistry, 2009. 284(23): p. 1535315357.

54. Dusterhoft, S., A. Babendreyer, A.A. Giese, C. Flasshove, et al., Status update on iRhom and ADAM17: It's still complicated. Biochim Biophys Acta Mol Cell Res, 2019. 1866(10): p. 15671583.

55. Jia, H.P., D.C. Look, P. Tan, L. Shi, et al., Ectodomain shedding of angiotensin converting enzyme 2 in human airway epithelia. Am J Physiol Lung Cell Mol Physiol, 2009. 297(1): p. L84-96.

56. Kozakov, D., D. Beglov, T. Bohnuud, S.E. Mottarella, et al., How good is automated protein docking? Proteins, 2013. 81(12): p. 2159-66.

57. Kozakov, D., D.R. Hall, B. Xia, K.A. Porter, et al., The ClusPro web server for protein-protein docking. Nat Protoc, 2017. 12(2): p. 255-278.

58. Vajda, S., C. Yueh, D. Beglov, T. Bohnuud, et al., New additions to the ClusPro server motivated by CAPRI. Proteins, 2017. 85(3): p. 435-444.

59. Schrodinger, L., The PyMOL molecular graphics system, version 2.4.0. 2020.

60. Baskaran, K., J.M. Duarte, N. Biyani, S. Bliven, et al., A PDB-wide, evolution-based assessment of protein-protein interfaces. BMC Struct Biol, 2014. 14: p. 22. 